\title{
A General Certification Framework with Applications to Privacy-Enhancing Certificate Infrastructures
}

\author{
Jan Camenisch, Dieter Sommer, and Roger Zimmermann \\ IBM Research, Zurich Research Lab \\ Säumerstrasse 4, CH-8803 Rüschlikon, Switzerland \\ \{jea, dso, zim\}@zurich.ibm.com
}

\begin{abstract}
Interactions in electronic media require mutual trust to be established, preferably through the release of certified information. Disclosing certificates for provisioning the required information often leads to the disclosure of additional information not required for the purpose of the interaction. For instance, ordinary certificates unnecessarily reveal their binary representation.

We propose a certificate-based framework comprising protocol definitions and abstract interface specifications for controlled, that is well-specified, release of data. This includes controlled release during the certification of data and controlled release of certified data. The protocols are based on proofs of knowledge of certificates and relations over the attributes, ensuring that no side information but only the specified data are revealed. Furthermore, the protocols allow one to release certified data in plain or encrypted form and to prove general expressions over the data items. Our framework can be seen as a generalization of anonymous credential systems, group signature, traceable signature, and e-cash schemes. The framework encompasses a specification language that allows one to precisely specify what data to release and how to release them in the protocols. We outline how our framework can be implemented cryptographically. The key application of our framework is the user-controlled release of attributes. Leveraging ideas of public key infrastructures, a privacy PKI (pPKI) can be built on top of the framework. We consider our framework a central building block to achieve privacy on the Internet.
\end{abstract}

\section{Introduction}

In today's interactions in electronic media users are frequently required to release personally identifying information (PII). A basic principle fostered by consumer protection agencies and privacy advocates is the idea of data minimization stating that the amount of data being provided should be minimal for a given purpose. Another principle is that users should be in control of their data.

The digital world makes it easy for organizations to build extensive profiles of users based on the data they obtain in interactions. It also provides new ways of protecting the privacy of users by employing cryptography to limit the amount of data being released in an interaction following the data minization principle.

Today's infrastructures within electronic media have often not been designed to take privacy into consideration. Scalable privacy support within such infrastructures 
requires the availability of a privacy architecture which allows for integrating privacy principles into the infrastructures. Research efforts involving privacy architecures are currently ongoing, for example, within the European PRIME project [28]. A privacy architecture includes privacy-enhancing authorization mechanisms allowing decisions to be based on a requester's (certified) attributes rather than on her identity, and languages for requesting general (certified) statements from a party. It is likely that such privacy architectures will emerge within the next few years.

In this paper, we define a general framework for data minimization allowing for precise specifications of what certified data to release to whom in a transaction and sketch the required cryptographic mechanisms. The full paper [13] gives an application of the framework to build a privacy-enhanced PKI (pPKI).

Our framework allows a party to obtain certificates while the issuer does not necessarily learn all data items being certified, but the issuer knows-through a proof performed by the party - that the party has a certificate from a particular issuer on such a data item. It furthermore allows the party to prove properties over certified data without revealing any other information, even not the certificate itself. The proofs encompass proofs of polynomial relations over certified data items and the computation of commitments and encryptions of polynomials over certified data items. The proofs allow for logical conjunction and disjunction operators over predicates on data items, encryptions, and commitments. The framework includes protocol descriptions with API definitions for obtaining certificates and proving knowledge and properties of certificates while being able to control precisely what data to release and to whom. We present a specification language for specifying this release of data.

Related Work. The research area our framework is positioned in was pioneered by Chaum who defined the concepts of credential systems, see for example [16], group signature schemes [19], and electronic cash systems [17]. Our framework can be seen as a generalization of these systems, as well as anonymous attestation schemes [5], traceable signature schemes [25] and identity escrow schemes [26]. Indeed, our framework can be instantiated to obtain a generalized anonymous credential system, a group signature scheme, traceable signature scheme, or an e-cash scheme. The pseudonym system of Brands [4] also provides efficient techniques for proving relations among committed values, but his overall construction falls short of supporting multi-show unlinkability thus restricting its applicability.

A cryptographic framework for releasing certified data has been proposed by Bangerter et al. [1]. The framework provides an initial step towards a general framework as put forth in our paper. The basic idea of using signature protocols for obtaining and proving signatures remains the same, but our approach is more general with respect to several aspects. It provides much more powerful proof capabilities like the proof of disjunctions, more general predicates and more functionality with respect to verifiable encryption. Moreover, our framework is more concrete in that we provide a specification language and concrete protocol interfaces close to an API-level making it ready for implementations and integration into privacy architectures and infrastructures. The high level of abstraction of our specification language allows for an easy integration into privacy architectures. The implementation of our framework is readily employable for pseudonymous authentication. 
Paper Outline. We first present our generalized framework for controlled data release in Section 2 including an interface specification for the protocols and the definition of a specification language and semantics for data release. Section 3 outlines the cryptographic instantiation of our framework. Section 4 concludes our work.

\section{A General Certification Framework Supporting Controlled Release of Data}

We define a general framework for the controlled release of certified data and controlled release of data for certification. Controlled release of certified data means that the releasing party can specify which information on data items of certificates to release and to whom. Controlled release of data for certification means that the issuer of a certificate learns only a specified subset of the data items being certified and for the remaining items only knows that particular parties have certified them for the certificate receiver.

Controlled release of certified data encompasses the release of (partial) information on the data items of certificates or polynomials over data items, either in clear or encrypted form. This involves computation of commitments and encryptions of certified data items or of multivariate polynomials.

Encryptions that contain a value that provably fulfills a multivariate polynomial relation between certified data items of multiple certificates, other commitments, and encryptions have a wide field of applications, such as conditional show of data, or escrow of values that provably fulfill some relation with respect to certified data items.

Our framework is based on Bangerter et al.'s framework [1], but offers more evolved functionality, in particular with respect to general proofs of conjunctions and disjunctions of predicates and a concrete specification language for specifying what information to release.

\subsection{Cryptographic Building Blocks}

The basic cryptographic building blocks of our framework are special signature schemes, commitment schemes, encryption schemes, and zero-knowledge proofs of knowledge.

Commitment Schemes. A commitment scheme allows a party to commit to a tuple $\left(x_{1}, \ldots, x_{u}\right)$ of (secret) values to another party. A commitment does not reveal any (computational) information on the tuple to the other party (hiding property) and prevents the committing party to change the values being committed to at a later stage (binding property). Either one of the properties can be information theoretic, but not both at the same time.

The Commit operation requires a public key $P K$ and a type specifier. In many schemes a random group element $r$ is created and returned as part of $o_{i}$.

$$
\left(c_{i}, o_{i}\right):=\operatorname{Commit}\left(x_{i, 1}, \ldots, x_{i, l}, P K\right)
$$

The object $c_{i}$ contains the information to be sent to the other party, namely the cryptographic commitment, and the public key. The object $o_{i}$ contains $x_{1}, \ldots, x_{u}$ and randomness $r$ and is retained by the committing party. If the commitment is used in a later proof, $o_{i}$ provides the secret information knowledge of which is proved and $c_{i}$ provides the corresponding information for the verifying party. 
Signature Schemes and Protocols. In our framework we make use of signature protocols for obtaining and proving knowledge of signatures on tuples of messages. Each message can either be known to the signer, he can know a commitment of it or have no information on it. Obtaining a signature is a protocol between the receiver of the signature and the issuer where the issuer learns only a subset of the messages to be signed. Proving knowledge of a signature on a tuple of messages is a protocol between a prover and a verifier where information on the messages $m_{i}$ is revealed selectively, i.e., allowing for proving relations on commitments to the messages $m_{i}$. Theoretically, such protocols can be obtained for any signature scheme, but these constructions would not be practical due to the required computational effort.

Two signature schemes with practical protocols, the SRSA-CL scheme [7] and the BL-CL scheme [8] are schemes that we use within our framework. These schemes allow proofs to be performed on the messages $m_{i}$ being signed. The schemes particularly allow that a signature be issued on messages that are not known to the issuer, but to which the issuer only knows a commitment. These schemes fit ideally into our framework as they allow the efficient discrete logarithm-based proofs of knowledge and relations between discrete logarithms of different certificates, commitments, and encryptions. For reasons of generality, schemes like RSA [29] fit into the framework as well, but without any of the privacy benefits of the above schemes.

Verifiable Encryption. Our framework makes use of encryption schemes to encrypt values within the protocol for proving knowledge of certificates. The encryption gets a tuple of messages $\left(m_{1}, \ldots, m_{k}\right)$, a label $L$, and a public key $P K E$ as input and outputs the encryption of the tuple.

The decryption algorithm requires a ciphertext, a label $L$, and a decryption key $S K E$ as input and outputs a tuple of messages $\left(m_{1}, \ldots, m_{k}\right)$. The label $L$ must be the same one as used for the encryption, it encodes a condition under which decryption may be performed and is cryptographically bound to the ciphertext. The key $S K E$ is private to a party trustworthy for a particular application. The party performs a decryption only in case the condition that is presented is fulfilled.

Within the certificate proof protocol properties over the messages $m_{i}$ of an encryption that has been computed can be proved, in particular polynomial relations to data items of certificates, commitments, and other encryptions.

The verifiable encryption schemes of Camenisch and Damgard [6] and of Camenisch and Shoup [11] are applicable to our framework. The latter are particularly suitable as they integrate smoothly into the discrete logarithm-based zero-knowledge proof protocols.

Zero-knowledge Proofs of Knowledge. In a zero-knowledge proof of knowledge a prover convinces a verifier that she knows a witness $w$ such that a predicate $P$ is fulfilled without releasing any further information on $w$. We employ zero-knowledge proofs for obtaining signatures and for proving knowledge of signatures. Within proof protocols, zero-knowledge proofs are used for proving knowledge of certificates, and proving relations between data items of certificates, commitments, and encryptions.

Concepts. A certificate on a tuple $\left(d_{1}, \ldots, d_{k}\right)$ of data items is a tuple $\left(d_{1}, \ldots, d_{k}, \sigma\right)$, where $\sigma$ is a signature on the data items. A certificate is obtained via an instance 
of the Certificatelssuance protocol. Knowledge of a certificate can be proved via the CertificateProof protocol. A certificate as defined in our framework allows one to treat data items individually in case the advanced signature protocols are being employed.

A certificate image or image is the result of a proof protocol involving one or multiple certificates. An image contains the assertion over the certificates that has been proved. We note that the image does-if our advanced signature protocols are usednot contain the signature of the issuer, but a transcript of a proof of knowledge of the signature performed by the owner. A proof can be made non-interactive by applying the Fiat-Shamir heuristics. This allows for a message to be signed. The signer is identified only by the properties being shown by the proof.

During the issuing protocol of a certificate, the signature on the data items is computed by the issuer without all data items being known to the issuer in clear and sent to the receiver. The proof protocol of a certificate proves to the verifier the prover's knowledge of a signature on data items while selectively revealing them or proving statements about them, and making commitments and encryptions of polynomials over data items. Multi-show unlinkability follows immediately from the fact the a certificate is not disclosed, but knowledge of it is proved using the appropriate cryptographic mechanisms.

We note that for the protocols defined below, certificates, commitments, and encryptions are defined on tuples of data items rather than on single items.

\subsection{Certificate Issuance Protocol}

The issuance of a certificate is a protocol Certificatelssuance between a receiver and an issuer. The outcome for the receiver is a certificate for the data items $d_{1}, \ldots, d_{k}$ of which the issuer only learns a subset. A proof involving the certificate can later be done with respect to the issuer's public key for signature verification, $P K S_{I}$.

A data item to be signed can either be known to the issuer in plaintext form, be known as a commitment to the data item, the data item can be jointly randomly generated, or it can be unknown to the issuer, i.e., known to the receiver only.

Known data items are used for all items that are contributed by the issuer or that the issuer has to know from a policy point of view. Committed data items are useful for the case where the receiver has proved before that the committed value is a particular data item appearing in another certificate and where this data item is to be included into the new certificate without the issuer learning it. An example for this is when the name and other personal data from a passport certificate should be included into the certificate to be issued. Jointly randomly generated data items are useful for limited-show certificates such as e-coins. Data items unknown to the issuer can be used to realize e-coins, as well.

During the execution of the protocol, the data items to be jointly randomly generated are computed and the issuer computes a value being (almost) a signature on the data items $d_{1}, \ldots, d_{k}$ and sends it to the receiver. The receiver obtains the final signature by tweaking the received signature by an easy computation with a value generated earlier in the protocol.

Protocol Interface Specification. The protocol Certificatelssuance for issuing a certificate is a protocol between a receiver and an issuer. Common input is presented to 
both parties and each party additionally receives private input. Both parties obtain commitments as output, the receiver in addition gets opening information to the commitments and the newly issued certificate as output. The certificate the receiver obtains is comprised of a tuple $\left(d_{1}, \ldots, d_{k}, \sigma\right)$ of data items $d_{i}$ and the signature $\sigma$ on the data items. The way the signature has been obtained for each individual data item is not reflected in the certificate.

Let $d_{1}, \ldots, d_{k}$ be the data items on which to obtain a certificate. Some of the items may be unknown as of the start of the protocol. Let $D=\{1, \ldots, k\}$ be the set of indices for the data items. Let the sets $D_{\text {known }}, D_{\text {committed, }} D_{\text {random, }}$ and $D_{\text {unknown }}$ partition $D$. Thus, $D=D_{\text {known }} \cup D_{\text {committed }} \cup D_{\text {random }} \cup D_{\text {unknown }}$ and the subsets are mutually disjoint. A data item $d_{i}$ with $i \in D_{\text {known }}$ is known to both parties, a data item $d_{i}$ with $i \in D_{\text {committed }}$ is known by the receiver only, the issuer has a commitment thereof. A $d_{i}$ with $i \in D_{\text {random }}$ is generated within the protocol, and a $d_{i}$ with $i \in D_{\text {unknown }}$ is known by the receiver, the issuer has no data regarding it. The interface of the protocol is specified as follows:

Certificatelssuance

\begin{tabular}{ll}
\hline Common input & $\left\{d_{i}: i \in D_{\text {known }}\right\},\left\{c_{i}: i \in D_{\text {committed }} \cup D_{\text {random }}\right\}, P K S_{I}, \chi$ \\
Receiver private input $\left\{o_{i}: i \in D_{\text {committed }} \cup D_{\text {random }}\right\},\left\{d_{i}: i \in D_{\text {unknown }}\right\}$ \\
Issuer private input & $S K S_{I}$ \\
\hline Receiver output & $\left\{c_{i}, o_{i}: i \in D_{\text {random }}\right\}$, cert \\
Issuer output & $\left\{c_{i}: i \in D_{\text {random }}\right\}$ \\
\hline
\end{tabular}

The commitments and openings $\left\{c_{i}, o_{i}: i \in D_{\text {random }}\right\}$ are uninstantiated when presented as protocol input and are generated during the protocol execution. The $o_{i} \mathrm{~s}$ contain the randomly generated data items for retrieval by the receiver and the $c_{i} s$ the corresponding commitments.

Any commitment $c_{i}$ contains--regardless of its instantiation state-information on the commitment public key, the algorithm, and the number of values in the tuple it will be a commitment of. After the protocol execution, the previously uninstantiated commitments and corresponding openings have been generated and the commitments can be retrieved by both parties, the opening information, however, on the receiver side only.

Issuance Specification Language. The issue specification $\chi$ passed in both party's inputs is expressed using a simple language specifying for each data item how it is contributed to the protocol and what information the issuer learns about it. This is defined by a triple for each data item: The first element is the index of the data item being specified where cert $[i]$ refers to $d_{i}$ of the certificate, the second element is a specifier of the issuance mode for the item being either of known, committed, random, or unknown. The last element is-depending on the second element-either a reference $d_{i}$ to a data item of the input, an index $c_{i}[j]$ to an instantiated commitment, an index to an uninstantiated commitment, or a reference $d_{i}$ to a data item depending on whether the second element specifies known, commitment, random, or unknown, respectively.

$$
\begin{array}{r}
\chi=\left\{\left(\operatorname{cert}[1], \text { known, } d_{1}\right),\left(\operatorname{cert}[2], \text { committed }, c_{1}[1]\right),\right. \\
\left.\left(\operatorname{cert}[3], \text { random, } c_{2}[1]\right),\left(\operatorname{cert}[4], \text { unknown, } d_{2}\right)\right\}
\end{array}
$$


The specification in Example (2) is for issuing a certificate on $\left(d_{1}, \ldots, d_{4}\right)$ where $D_{\text {known }}=\{1\}, D_{\text {committed }}=\{2\}, D_{\text {random }}=\{3\}$, and $D_{\text {unknown }}=\{4\}$. After successful protocol execution, $o_{3}[1]$ contains the randomly generated data item $d_{3}$ and $c_{3}[1]$ contains a commitment of $d_{3}$.

\subsection{Certificate Proof protocol}

The protocol CertificateProof is a protocol between a prover and a verifier that allows the prover to prove to the verifier knowledge of valid certificates and general statements over the data items of multiple certificates, commitments, and encryptions. It further allows one to compute commitments and encryptions specified through multivariate polynomials over data items, encryptions, and commitments.

The underlying key principle is that certificates are not sent, but statements regarding them are proved using zero-knowledge proofs of knowledge such that no more information than what is specified is revealed. From the applied cryptographic mechanisms it follows immediately that multiple proofs over the same certificate are mutually unlinkable and unlinkable to the Certificatelssuance protocol instance it was issued in.

Protocol Interface Specification. The common inputs to the prover and verifier are the public keys for signature verification $P K S_{I_{1}}, \ldots, P K S_{I_{k}}$, (possibly uninstantiated) commitments $c_{1}, \ldots, c_{u}$, and (possibly uninstantiated) encryptions $e_{1}, \ldots, e_{v}$. Let the sets $\hat{C} \subseteq C=\{1 \ldots, u\}$ and $\hat{E} \subseteq E=\{1, \ldots, v\}$ contain the indices of uninstantiated commitments and uninstantiated encryptions, respectively. The prover additionally gets as input a list of certificates cert $_{1}, \ldots$, cert $_{k}$ having been obtained from issuers $I_{1}, \ldots, I_{k}$, (uninstantiated) opening information $o_{1}, \ldots, o_{u}$ corresponding to the commitments, and a prover-side proof specification $\xi_{P}$. The verifier gets a verifier-side proof specification $\xi_{V}$ as input.

The verifier's output are the commitments $\left\{c_{i}: i \in \hat{C}\right\}$ and encryptions $\left\{e_{i}: i \in\right.$ $\hat{E}\}$ having been computed during the protocol execution and a certificate image $\Pi$ containing the statement conveyed through the proof together with the proof transcript. The prover's output are the commitments and openings $\left\{c_{i}, o_{i}: i \in \hat{C}\right\}$ and the encryptions and corresponding plaintexts $\left\{e_{i}, p_{i}: i \in \hat{E}\right\}$.

CertificateProof

Common input $P K S_{I_{1}}, \ldots, P K S_{I_{k}}, c_{1}, \ldots, c_{u}, e_{1}, \ldots, e_{v}$

Prover private input $\operatorname{cert}_{1}, \ldots, \operatorname{cert}_{k}, o_{1}, \ldots, o_{u}, p_{1}, \ldots, p_{v}, \xi_{P}$

Verifier private input $\xi_{V}$

\begin{tabular}{ll}
\hline Prover output & $\left\{c_{i}, o_{i}: i \in \hat{C}\right\},\left\{e_{i}, p_{i}: i \in \hat{E}\right\}$ \\
Verifier output & $\left\{c_{i}: i \in \hat{C}\right\},\left\{e_{i}: i \in \hat{E}\right\}, \Pi$ \\
\hline
\end{tabular}

Commitments. The set $C=\{1, \ldots, u\}$ defines the indices $i$ of the commitments and openings: $\left\{c_{i}, o_{i}: i \in C\right\}$. The subset of the commitments and corresponding openings $\left\{c_{i}, o_{i}: i \in \hat{C} \subseteq C\right\}$ are uninstantiated, i.e., the commitment contains only information on the commitment public key and further parameters, but not yet a cryptographic commitment and the openings contain not yet the opening information for the commitments. The objects $\left\{c_{i}, o_{i}: i \in C \backslash \hat{C}\right\}$ are instantiated commitments and openings. 
Uninstantiated commitments in the proof protocol allow one to create commitments during the protocol execution that can later be used to issue a signature on the value being committed to or to use it within other proofs. An application example for this is if an organization issues a certificate that shall contain the user's identity from another certificate. To achieve this, the other certificate is first proved and the identity data item is being committed to. Then the new certificate is issued using that commitment and other data items.

Encryptions. Analogously, the set $E=\{1, \ldots, v\}$ defines the indices for the encryptions $e_{i}$ and corresponding plaintexts $p_{i}$. The subset $\hat{E} \subseteq E$ defines again the set of uninstantiated encryptions and plaintexts. An uninstantiated encryption contains an algorithm identifier, a label $L$, and an encryption public key $P K E_{T}$ of a party $T$, and the number of elements in the plaintext tuple of the encryption. The label $L$ defines a condition under which party $T$ will perform a decryption. The same label that has been used for encryption and a private key $S K E_{T}$ known to $T$ has to be passed to the decryption algorithm in order for the algorithm to decrypt correctly. Considering these properties, the encryptions realize a conditional show of the underlying values under condition $L$.

Uninstantiated encryptions will be computed during the protocol execution. After the protocol has terminated, the encryptions and corresponding plaintexts can be retrieved, plaintexts only by the prover.

Proof Specification. The proof specification defines what statement to prove over the list of certificates, commitments, and encryptions, thereby also defining what values to commit to and what values to encrypt. The proof specification $\xi_{P}$ for the prover and $\xi_{V}$ for the verifier are very similar, the only difference being that the prover's specification in addition contains a language element to specify what parts of a disjunction to prove. The specification language will be further elaborated on below.

Specification Language. A proof specification $\xi_{P}$ or $\xi_{V}$ is a logical formula in propositional logics without negation over a class of predicates defined below.

Variables. The predicates in a formula refer to variables to reference data items, certificates, committed values, and encrypted values of the input. We stress that a variable always references the secret input of the prover and is used for expressing a statement over this secret input. Secret input are certificates, commitment opening information, and plaintexts to encryptions. The verifier only has corresponding data objects that do not allow to infer the secrets. Such objects are commitments or encryptions.

Referenced commitments and encryptions and their corresponding opening and plaintext objects can be either uninstantiated or instantiated. Data items are references to the (secret) data items of certificates expressed with variables of the form cert ${ }_{i}[j]$ where $i$ is the index of the certificate the data item is contained in and $j$ is the index within the data items of the certificate. This notation allows to have multiple certificates of the same type, e.g. bank statements being used. A variable $c_{i}[j]$ refers to the $j$-th value of the tuple of values committed to with commitment $i$. A variable $e_{i}[j]$ refers to the $j$-th plaintext value corresponding to the tuple of values being the plaintext for the $i$-th encrypted tuple. If a $c$ or $e$ refers to an uninstantiated instance, restrictions are imposed on the usage of the variable. 
Predicates. A predicate is composed of either a term or a comparison operator and two terms as arguments. A term is either an atom or an expression. An atom is either a variable or a constant. An expression is either an arithmetic operator applied to expressions, or an atom. Parantheses can be used to override standard precedence.

Multivariate polynomial arithmetic relations are defined over an interval in $\mathbb{Z}$, e.g. $\left[-2^{256}+1 ; 2^{256}-1\right]$, with operators being "+" and ".". The exponentiation operator is expressed as usual. ${ }^{1}$ The usual semantics applies for all operators over the integers. The relational operator in a predicate can be any of $=, \neq,\rangle,\langle, \geqslant$, or $\leqslant$ with their usual semantics in the integers. All results of expressions must be within the integer interval defined through the system parameters.

$$
\begin{array}{lll}
\operatorname{cert}_{1} & (3) & \operatorname{cert}_{1}[1] \geqslant c_{1}[2] \\
\operatorname{cert}_{1}[1] & (4) & 10 \cdot \operatorname{cert}_{1}[1]+20 \cdot \operatorname{cert} t_{2}[1]^{4}=\operatorname{enc}_{1}[1]+40
\end{array}
$$

Example (3) expresses knowledge of the signature of certificate cert 1 . Example (3) is equivalent to Example (4). Example (4) states knowledge of a signature of the data item $d_{1}$ of cert $_{1}$ without any further statement. It implies knowledge of the certificate. In Example (5) a greater-than-or-equal relation is stated between the data item $d_{1}$ in cert $t_{1}$ and the second element of the tuple committed to with $c_{1}$. Example (6) states a more general polynomial relation.

Specification Formula. The specification formula is a formula in propositional logics without negation and with predicates as defined above. Thus the logical operators are the conjunction $(\wedge)$ and disjunction $(V)$ operators. The usual precedence and semantics of the operators applies. Parantheses can be applied in their usual semantics for changing precedence. It is conceivable that an XML-based corresponding prefix notation instead of the infix notation used here be used in practice due to easier processing.

We introduce the \langle\rangle language element to allow a prover to specify what predicates of a formula to fulfill with secrets provided as input. This is required as a logical formula can allow that multiple different clauses in its disjunctive normal form (DNF) be fulfillable by the certificates provided as input, for example both predicates in the formula $\operatorname{cert}_{1}[1]=10 \vee \operatorname{cert}_{1}[2]=20$ can be fulfilled with one certificate cert $_{1}$ with appropriate data items. One pair of \langle\rangle elements can span multiple $\wedge$-connected predicates. When constructing the DNF of a formula, the \langle\rangle -annotation propagates to the DNF representation with the predicates it spans. It is important for the semantics of instantiation of commitments and encryptions that in exactly one clause of the DNF all predicates have a \langle\rangle annotation. The \langle\rangle notation is, of course, only applied to the prover's specification. The remainder of the proof specification is equal for the prover and verifier in the protocol.

$$
\left\langle\operatorname{cert}_{1}[1]>21\right\rangle \vee \operatorname{cert}_{2}[1]
$$

Example (7) specifies that the certified data item cert , $_{1}[1]$ is greater than 21 or that the prover knows a signature on data item cert $_{2}[1]$. It evaluates to true if either of the predicates is fulfilled. The \langle\rangle notation defines that the prover provides $c e r t_{1}$ as input.

\footnotetext{
${ }^{1}$ Processing of exponents requires additional runtime linear in the length of an efficiently computable addition chain for the exponent.
} 
Uninstantiated Variables. Instantiated variables (encryptions and commitments) are instantiated through the presented certificates, commitments, and encryptions. Uninstantiated commitments are instantiated as a first step in the protocol and uninstantiated encryptions are computed. Both are specified by the \langle\rangle -annotated predicates they appear in when considering the DNF of the formula. Predicates annotated with \langle\rangle must be consistent to each other in defining variables' values. Predicates not within 〈> can make different statements about the variables. When a commitment or encryption is not defined by \langle\rangle predicates, it is initialized with a random value. After the instantiation phase all committed and encrypted values are fixed.

The actual instantiation of the variable and its underlying commitment or encryption is governed by the prover by the \langle\rangle language element. Thus one can think of it being correctly instantiated when referring to it in predicates.

$$
\begin{aligned}
& c_{1}[1]=\operatorname{cert}_{1}[1]+\operatorname{cert}_{2}[1]^{2} \\
& \operatorname{cert}_{1}[1]+\operatorname{cert}_{1}[2]=\operatorname{cert}_{1}[3]+c_{1}[1] \\
& \left(\operatorname{cert}_{1}[1]>10 \wedge e_{1}[1]=\operatorname{cert}_{1}[1]\right) \vee\left\langle\left(\operatorname{cert}_{1}[1] \leqslant 10 \wedge e_{1}[1]=\operatorname{cert}_{1}[2]\right)\right\rangle \\
& \left\langle\left(\operatorname{cert}_{1}[1]=10\right)\right\rangle \vee\left(\operatorname{cert}_{1}[1]=20 \wedge e_{1}[1]=\operatorname{cert}_{1}[2]\right)
\end{aligned}
$$

The uninstantiated variable $c_{1}[1]$ gets assigned cert $t_{1}[1]+\operatorname{cert}_{2}[1]^{2}$ in example (8). In example (9), the uninstantiated variable $c_{1}[1]$ gets assigned cert $_{1}[1]+$ cert $_{1}[2]-$ cert $_{1}[3]$. In Example (10) above, $e_{1}[1]$ is instantiated with cert $t_{1}[2]$. In Example (11) $e_{1}[1]$ is instantiated with a random number.

Semantics of the Specification. The semantics of propositional logics without negation, like that of the logical operators, and of the defined class of predicates translates directly into the semantics of a specification formula. The semantics of the variables is defined by using the set of the prover's certificates and the provided encryptions and commitments as the universe to interpret the formula.

The entailment semantics (formal semantics) of a specification formula is that of propositional logics without negation with the class of predicates as defined. This formal semantics is, for example, required for automated reasoning over a valid proof specification. An application of this is computing an access decision.

Certificate Images. Depending on the way the proof is performed cryptographically, the certificate image can be either convincing to third parties or does not contain any information on whether the proof was actually executed. We denote the further a transferable image and the latter a non-transferable image. The transferable image is based on a noninteractive proof of knowledge [23] and has much the same properties as a conventional certificate, i.e., it is a statement about its owner endorsed by an issuing party. In fact, a transferable image can be endorsed by multiple parties in case certificates of different issuers were involved in the proof. This concept of a certificate containing conjunctions and disjunctions of predicates over data items of different issuers is a new concept. It is interesting to note that this certificate is created within a proof protocol which did not involve any issuers and nevertheless is a statement endorsed by all these issuers. Thus, a transferable certificate image of a certificate in our framework much resembles the classical idea of a certificate. Additionally, a message can be signed with a transferable 
image, the signer being identified through the proved statement only. This allows new applications of certifying, for example, public keys.

Example for Using our Framework. When considering current directions of legislation towards preventing unconditional anonymity, use cases based on conditional anonymity become increasingly important. Consider, for example, a service provider being required to provide - under well-defined conditions such as a court order-an identityequivalent item of the service requester for each transaction. Assume existence of a US authority and a European Union authority each providing a public key for encryption. Consider furthermore that users have electronic passport certificates issued either by the US or the European Union. Let the certificate cert $_{1}$ in the example be a US passport, cert $_{2}$ a European Union passport. Assume a user has only a US passport. Let the data item $d_{1}[1]$ be the passport number in the passports which, if known to the respective authority, easily allows to find the associated holder.

$$
\begin{aligned}
& \left\langle\left(e_{1}[1]=\operatorname{cert}_{1}[1] \wedge e_{1}[2]=1 \wedge e_{2}[2]=0\right)\right\rangle \vee \\
& \left(e_{2}[1]=\operatorname{cert}_{2}[1] \wedge e_{2}[2]=1 \wedge e_{1}[2]=0\right)
\end{aligned}
$$

Example 12 states either of the following: $i$ ) The prover has a United States passport and its serial number is encrypted in $e_{1}[1]$, the constant 1 is encrypted in $e_{1}[2]$ indicating that $e_{1}$ is the encryption containing a valid passport number. For encryption the public key of the US authority and an appropriate $L_{U S}$ (both provided in the input) is used. Additionally, a 0 is encrypted in $e_{2}$ [2] indicating that $e_{2}$ contains no passport number. For the latter, the EU public key and EU condition $L_{E U}$ is used. No statement is made regarding $e_{2}$ [1]. ii) The user has a European Union passport and this passport's serial number's encryption is $e_{2}[1]$ and the constant 1 is encrypted in $e_{2}[2]$ indicating that $e_{2}$ contains the user's passport number. This is done using the encryption key of the EU agency and a condition $L_{E U}$. A 0 is encrypted under the US key and condition in $e_{1}$ [2]. No statement is made regarding $e_{1}[1]$.

By using this proof of a disjunction, no information on which passport the user has is conveyed. Only in the rare case when the encryptions have to be decrypted by the respective government agency under the defined condition, will it also be clear what country the user is a citizen of.

\section{Cryptographic implementation}

In the common parameters model we build upon several known protocols for proving statements about discrete logarithms, such as (1) proof of knowledge of a discrete logarithm modulo a prime [30] or a composite [24,22], (2) proof of knowledge of equality of representation modulo two (possibly different) prime [18] or composite [10] moduli, (3) proof that a commitment opens to the product of two other committed values $[9,14$, $3]$, (4) proof that a committed value lies in a given integer interval $[15,9,2]$, and also (5) proof of the disjunction or conjunction of any two of the previous [20].

That is, we can use these protocol to efficiently prove the statements we defined among messages that are (1) committed using the Pedersen [27] or Damgard-Fujisaki 
commitment schemes [22], (2) encrypted using the Camenisch-Shoup encryption scheme, or (3) signed using the Camenisch-Lysyanskaya signature schemes $[7,8]$ without revealing the messages themselves. For obtaining concurrent zero-knowledge proofs, Damgård's construction [21] is applied. Non-interactive zero-knowledge proofs are obtained by using the Fiat-Shamir heuristic [23]. The latter in addition allows one to create a group signature scheme of the protocol for proving certificates. The detailed protocol specifications are to appear in a research report [12].

\section{Conclusion}

We defined a general certificate framework for obtaining certificates and (partially) releasing certified data either in plain or encrypted form. In particular, our framework is a step to realize proactive privacy. The framework includes cryptographic primitives for realizing the functionality, definition of protocol interfaces for the Certificatelssuance and CertificateProof protocols, and a powerful specification language with using semantics of propositional logics that allows a prover to precisely specify what data to release in a transaction. We briefly outline our implementation efforts. In the full paper [13] we explain how to apply our framework to define privacy extensions to the current Internet certificate infrastructure. Our contributions can pave the way to deploying long-needed privacy technology within communication networks such as the Internet.

\section{Acknowledgements}

Part of the work reported in this paper is supported by the European Commission by the IST Project PRIME. The PRIME project receives research funding from the European Community's Sixth Framework Programme and the Swiss Federal Office for Education and Science.

\section{References}

1. Bangerter, E., Camenisch, J., and Lysyanskaya, A. A cryptographic framework for the controlled release of certified data. In Twelfth International Workshop on Security Protocols 2004 (2004), LNCS, Springer Verlag.

2. Boudot, F. Efficient proofs that a committed number lies in an interval. In EUROCRYPT 2000, vol. 1807 of LNCS, Springer Verlag, pp. 431-444.

3. Brands, S. Rapid demonstration of linear relations connected by boolean operators. In $E U$. ROCRYPT '97 (1997), vol. 1233 of $L N C S$, Springer Verlag, pp. 318-333.

4. Brands, S. Rethinking Public Key Infrastructure and Digital Certificates-Building in Privacy. $\mathrm{PhD}$ thesis, Eindhoven Institute of Technology, Eindhoven, The Netherlands, 1999.

5. Brickell, E., Camenisch, J., and Chen, L. Direct anonymous attestation. In $A C M C C S$ '04, ACM Press, pp. 132-145.

6. Camenisch, J., and Damgård, I. Verifiable encryption, group encryption, and their applications to group signatures and signature sharing schemes. In ASIACRYPT 2000, vol. 1976 of LNCS, Springer Verlag, pp. 331-345.

7. Camenisch, J., and Lysyanskaya, A. A signature scheme with efficient protocols. In Third Conference on Security in Communication Networks (2002), vol. 2576 of LNCS, Springer Verlag, pp. 274-295.

8. Camenisch, J., and Lysyanskaya, A. Signature schemes and anonymous credentials from bilinear maps. In CRYPTO 2004, Springer Verlag. 
9. Camenisch, J., and Michels, M. Proving in zero-knowledge that a number $n$ is the product of two safe primes. In EUROCRYPT '99, vol. 1592 of $L N C S$, Springer Verlag, pp. 107-122.

10. Camenisch, J., and Michels, M. Separability and efficiency for generic group signature schemes. In CRYPTO '99 (1999), vol. 1666 of LNCS, Springer Verlag, pp. 413-430.

11. Camenisch, J., and Shoup, V. Practical verifiable encryption and decryption of discrete logarithms. In CRYPTO 2003, LNCS.

12. Camenisch, J., and Sommer, D. Tech. Rep. Research Report RZ 3646, IBM Zurich Research Laboratory, 2006.

13. Camenisch, J., Sommer, D., and Zimmermann, R. A general certification framework with applications to privacy-enhancing certificate infrastructures. Tech. Rep. 3629, IBM Zurich Research Laboratory, November 2005.

14. Camenisch, J. L. Group Signature Schemes and Payment Systems Based on the Discrete Logarithm Problem. PhD thesis, ETH Zürich, 1998. Diss. ETH No. 12520, Hartung Gorre Verlag, Konstanz.

15. Chan, A., Frankel, Y., and Tsiounis, Y. Easy come - easy go divisible cash. In EUROCRYPT '98 (1998), vol. 1403 of LNCS, Springer Verlag, pp. 561-575.

16. Chaum, D. Untraceable electronic mail, return addresses, and digital pseudonyms. Communications of the ACM 24, 2 (Feb. 1981), 84-88.

17. Chaum, D. Blind signature systems. In CRYPTO '83, Plenum Press, p. 153.

18. Chaum, D., and Pedersen, T. P. Wallet databases with observers. In CRYPTO '92, vol. 740 of $L N C S$, Springer-Verlag, pp. 89-105.

19. Chaum, D., and van Heyst, E. Group signatures. In EUROCRYPT '9I (1991), vol. 547 of LNCS, Springer-Verlag, pp. 257-265.

20. Cramer, R., Damgård, I., and Schoenmakers, B. Proofs of partial knowledge and simplified design of witness hiding protocols. In CRYPTO '94 (1994), vol. 839 of LNCS, Springer Verlag, pp. 174-187.

21. Damgård, I. Efficient concurrent zero-knowledge in the auxiliary string model. In EUROCRYPT 2000, vol. 1807 of LNCS, Springer Verlag, pp. 431-444.

22. Damgård, I., and Fujisaki, E. An integer commitment scheme based on groups with hidden order. In $A S I A C R Y P T$ 2002, vol. 2501 of LNCS, Springer.

23. Fiat, A., and Shamir, A. How to prove yourself: Practical solutions to identification and signature problems. In CRYPTO '86, vol. 263 of LNCS, Springer Verlag, pp. 186-194.

24. Fujisaki, E., and Okamoto, T. Statistical zero knowledge protocols to prove modular polynomial relations. In CRYPTO '97, vol. 1294 of LNCS, Springer Verlag, pp. 16-30.

25. Kiayias, A., Tsiounis, Y., and Yung, M. Traceable signatures. In EUROCRYPT, vol. 3027 of LNCS, Springer, pp. 571-589.

26. Kilian, J., and Petrank, E. Identity escrow. Theory of Cryptography Library, Record Nr. 97-11, http://theory. lcs.mit .edu/ tcryptol, Aug. 1997.

27. Pedersen, T. P. Non-interactive and information-theoretic secure verifiable secret sharing. In CRYPTO '91, vol. 576 of LNCS, Springer Verlag, pp. 129-140.

28. PRIME project. Www . prime-project . eu.org.

29. Rivest, R., Shamir, A., and Adleman, L. A method for obtaining digital signatures and public-key cryptosystems. Communications of the ACM 21, 2 (Feb. 1978), 120-126.

30. Schnorr, C. P. Efficient signature generation for smart cards. Journal of Cryptology 4,3 (1991), 239-252. 